\title{
Letter to the Editor-in-Chief
}

Fibrin monomers derived from thrombogenic dysfibrinogenemia, Naples-type variant (BßAla68Thr), showed almost entirely normal polymerization

\section{Tomu Kamijo $^{1}$, Kazuhiro Nagata ${ }^{2}$, Chiaki Taira $^{1}$, Yumiko Higuchi ${ }^{1}$, Shinpei Arai ${ }^{3}$, Nobuo Okumura ${ }^{1}$}

1) Department of Clinical Laboratory Investigation, Graduate School of Medicine, Shinshu University, Matsumoto, Japan, 2) Department of Clinical Laboratory, Minami-Nagano Medical Center, Shinmachi Hospital, Nagano, Japan, and 3) Department of Laboratory Medicine, Shinshu University Hospital, Matsumoto, Japan.

Key Words: dysfibrinogenemia, thrombosis, fibrin polymerization, fibrinopeptide

release

Word count: 1406, excluding the title page, references, table, figure, and figure legend.

Correspondence to: Yumiko Higuchi, PhD, Department of Biochemical Laboratory

Sciences, School of Health Sciences,

Shinshu University, 3-1-1 Asahi, Matsumoto 390-8621, Japan

Phone: (81) 26337 2387; Fax: (81) 26337 2370;

E-mail: sasa0922@shinshu-u.ac.jp 
Inherited fibrinogen disorders causing quantitative or qualitative alterations in this molecule have been classified phenotypically as hypofibrinogenemia, afibrinogenemia, dysfibrinogenemia, and hypodysfibrinogenemia. As many as 400 cases of inherited fibrinogen disorders have been listed on the GEHT homepage [1]. The clinical manifestations of inherited fibrinogen disorders are very heterogeneous: the absence of symptoms, bleeding, thrombosis, and recurrent miscarriages in women as well as rare cases of fibrinogen storage disease and renal amyloidosis. Approximately $14 \%$ of reported dysfibrinogens are associated with thrombosis [2]; however, difficulties are associated with demonstrating that fibrinogen mutations directly cause thrombosis. The homozygous BßAla68Thr mutation, known as Naples I or Milano II [3], which is one of the most common mutations associated with thrombosis, causes markedly defective thrombin binding to its fibrin in vitro and an increase in free active thrombin in the circulation [4]. Zhou J et al. reported the same mutation in five Chinese patients, two of whom had thrombosis [5].

We recently identified homozygous dysfibrinogenemia, a BßAla68Thr variant, in a 17-year-old Japanese boy who developed superior sagittal sinus thrombosis [6]. We herein examined the thrombin-associated functions of fibrinogen purified from the plasma of this homozygous patient, and compared them with those of fibrinogen from a 
heterozygous patient and normal control.

This study was approved by the Ethics Review Board of Shinshu University School of Medicine (approved number 603). After informed consent had been obtained from the propositus and his family members, blood samples were collected for biochemical and genetic analyses. Coagulation tests, DNA sequencing, the purification of plasma fibrinogen, kinetics of fibrinopeptide A (FpA) and fibrinopeptide B (FpB) release, and fibrin polymerization were examined as previously described [7-9], with minor modifications.

The coagulation test results of plasma collected from the propositus, his parents, and two healthy sisters are shown in Table 1 . The plasma fibrinogen concentration ratios (the thrombin time method/immunological method) of the propositus and his younger sister were 0.369 and 0.364 , respectively, and were lower than those of the others $(0.594$ 0.671). Moreover, the thrombin clotting times of the propositus' and his younger sister's plasma were 90.0 and 118.0 sec, respectively, and were longer than those of the others (20.6 24.5). Plasma levels of protein C, protein S, antithrombin, plasminogen lupus anticoagulant, antiphospholipid antibodies, and homocysteine in the propositus were within normal ranges, but were not measured in his younger sister's plasma [6]. A DNA sequence analysis of FGA, FGB, and FGG for the propositus and his 
younger sister revealed a homozygous G>A mutation in exon 2 of FGB (c.292 G>A;

NCBI NM_005411), resulting in a substitution of Bß68Ala (GCT) > Thr (ACT) in the mature protein (or Bß98T with the signal peptide), while the others were heterozygotes for this mutation. This mutation is known as fibrinogen Naples, which causes thrombogenic dysfibrinogenemia in homozygous patients, but not in heterozygous patients.

The rate of thrombin-catalyzed FpA release at an ambient temperature from the propositus' fibrinogen was markedly slower than that from normal control fibrinogen, while that from the father's fibrinogen was slightly slower (Fig. 1A). Furthermore, the rate of thrombin-catalyzed $\mathrm{FpB}$ release from the propositus' fibrinogen was markedly slower, while that from the father’s fibrinogen was slightly slower (Fig. 1B). However, the rates of batroxobin-catalyzed FpA release from the propositus' and father's fibrinogen were similar to that from normal control fibrinogen (data not shown).

Representative curves of thrombin-catalyzed fibrin polymerization and fibrin monomers performed at an ambient temperature are shown in Figure 1C-1F. The propositus’ fibrinogen showed a longer lag period and markedly decreased thrombin-catalyzed fibrin polymerization than the normal control under the conditions of $0.18 \mathrm{mg} / \mathrm{mL}$ of fibrinogen and $0.05 \mathrm{U} / \mathrm{mL}$ of thrombin. The heterozygous father's 
fibrinogen showed a slightly longer lag period and a decreased rate of

thrombin-catalyzed fibrin polymerization than the control (Fig. 1C). However,

batroxobin-catalyzed fibrin polymerization in the propositus and his father was similar

to that of the normal control under the conditions of $0.18 \mathrm{mg} / \mathrm{mL}$ of fibrinogen and 0.05

$\mathrm{U} / \mathrm{mL}$ of batroxobin (data not shown).The polymerization of the propositus' fibrinogen

was more than two-fold the maximum slope of the reaction curve and there was an

absorbance change at 60 minutes at $0.36 \mathrm{mg} / \mathrm{dL}$ of fibrinogen, relative to $0.18 \mathrm{mg} / \mathrm{dL}$

(Fig. 1D). Furthermore, under the condition of $1.0 \mathrm{U} / \mathrm{mL}$ of thrombin, the

polymerization of the propositus' fibrinogen showed a 16-fold shorter lag period and steeper slope than that under the condition of $0.05 \mathrm{U} / \mathrm{mL}$ of thrombin (Fig. 1E).

In order to analyze the polymerization ability of homozygous variant fibrinogen, fibrin monomers derived from a thrombin-initiated clot were re-polymerized in $\mathrm{pH} 7.4$ buffer. In contrast to our expectations, fibrin monomers derived from a homozygous variant fibrin clot were re-polymerized in a similar manner to those from the normal control (Fig. 1F). Furthermore, in order to examine the marked differences observed in thrombin-catalyzed polymerization, we measured clottability after a 5-hour incubation at $37^{\circ} \mathrm{C}$. Clottability was $97.4 \pm 1.3 \%$ for normal control fibrinogen. The corresponding values of the propositus' and father's fibrinogen were $87.8 \pm 3.1 \%(p<0.01)$ and $84.5 \pm$ 
$2.3 \%(p<0.005)$, respectively.

We observed fibrin clots using scanning electron microscopy in order to analyze differences in the ultrastructures of fibrin clots formed at $37^{\circ} \mathrm{C}$ from the fibrinogen of the normal control, propositus, and his father. Although the density of the bundles of fibrin fibers was less in the order of clots made from fibrinogen of the normal control, the father (heterozygote), and the propositus (homozygote) (photo not shown), fiber diameters were not significantly different between them (data not shown).

We herein identified homozygous dysfibrinogenemia, BßAla68Thr, which manifested as severe thrombosis. A family study revealed one homozygous and three heterozygous members, none of whom exhibited thrombotic complications. The same variant in an asymptomatic heterozygous patient was previously reported as fibrinogen Shizuoka, a resident in the same city as the present propositus (Yonekawa O. et al., $16^{\text {th }}$ Congress of International Society on Thrombosis and Haemostasis, Florence, 1997, PS-2550). Three homozygous patients of the B $\beta$ Ala68Thr fibrinogen family (Naples I or Milano II) manifested thrombosis: two developed strokes at 25 and 21 years of age, respectively, while the other developed DVT after surgery [3]. The markedly defective thrombin binding of homozygous B $\beta$ Ala68Thr fibrin was demonstrated in vitro as the thrombosis-inducing mechanism, suggesting an increase in free active thrombin in the 
circulation of the homozygous B $\beta$ Ala68Thr patient [4]. Zhou J et al. reported that five patients from one Chinese family had the same variants; two out of the five patients had thrombosis, and it was unclear whether two patients were a homozygote or heterozygote $[5]$.

The homozygous B $\beta A$ la68Thr variant also showed a markedly prolonged thrombin clotting time, lower fibrinogen ratio (functional method/immunological method), and markedly reduced release rate of FpA and $\mathrm{FpB}$ by thrombin. In contrast, the heterozygous B $\beta$ Ala68Thr variant showed a slightly prolonged thrombin clotting time, slightly lower fibrinogen ratio, and slightly reduced release rate of FpA and FpB by thrombin. The thrombin-catalyzed fibrin polymerization of homozygous B $\beta A l a 68 T h r$ fibrinogen was markedly less than that of the normal control (longer lag period and less steep slope), whereas fibrin monomers derived from the homozygous variant fibrin clot were re-polymerized in a similar manner to those from the normal control. The markedly reduced thrombin-catalyzed fibrin polymerization of homozygous BßAla68Thr fibrinogen was caused by the markedly delayed release of FpA and FpB. However, once FpA and FpB were released and fibrin monomers were produced, the substitution of Thr for B $\beta$ Ala68 did not affect fibrin monomer polymerization. This phenomenon was markedly different from the aberrant polymerization of fibrin 
monomers derived from the thrombin-catalyzed fibrin of recombinant variant BßGly15Cys- and BßGly15Ala-fibrinogen [10]. These findings demonstrate that the $\mathrm{B} \beta$ Ala68 residue is not important for the lateral aggregation of desAB fibrin monomer polymerization.

Homozygous B $\beta A l a 68 T h r$ fibrinogen showed markedly aberrant thrombin-catalyzed fibrin formation at an ambient temperature, but also nearly normal thrombin-induced fibrinogen clottability and clot structures (and fiber diameters) in a $37^{\circ} \mathrm{C}$ reaction. We speculate that a longer incubation at $37^{\circ} \mathrm{C}$ may enhance the thrombin-catalyzed fibrin polymerization of homozygous B $\beta$ Ala68Thr fibrinogen. Therefore, the fibrin conversion ability of the fibrinogen of homozygous patients may not be severely reduced in vivo and the risk of bleeding may not be very high.

\section{Disclosure of Conflict of Interests}

The authors state that they have no conflicts of interest.

\section{Acknowledgments}

We gratefully acknowledge Dr. Kenji Nara and Koji Amano (Seirei-Mikatabara General Hospital, Hamamatsu, Shizuoka Pref.) for the patient referrals. This work was supported 
by JSPS KAKENHI Grant Number JP17K09009 (Chiaki Taira and Nobuo Okumura).

\section{References}

[1] Groupe d’Etude sur 1’Hémostase et la Thrombose. "Base de données des variants du Fibrinogène”. GEHT Web site, < http://site.geht.org/base-de-donnees-fibrinogene/ > (updated on 28/09/2017) .

[2] A. Casini, M. Blondon, A. Lebreton, J. Koegel, V. Tintillier, E. de Maistre, P. Gautier, C. Biron, M. Neerman-Arbez, P. de Moerloose, Natural history of patients with congenital dysfibrinogenemia, Blood 125(2015) 553-561.

[3] F. Haverkate, J. Koopman, C. Kluft, A.D’Angelo, M. Cattaneo, P.M. Mannucci, Fibrinogen Milano II: A congenital dysfibrinogenemia associated with juvenile arterial and venous thrombosis, Thromb. Haemost.55 (1986) 131-135.

[4] J. Koopman, F. Haverkate, S.T. Lord, J. Grimbergeb, P.M. Mannucci, Molecular basis of fibrinogen Naples associated with defective thrombin binding and thrombophilia, J. Clin. Invest. 90(1992) 238-244.

[5] J. Zhou, Q. Ding, Y. Chen, Q. Ouyang, L. Jiang, J. Dai, Y. Lu, X. Wu, Q. Liang, H. Wang, X. Wang, Clinical features and molecular basis of 102 Chinese patients with congenital dysfibrinogenemia, Blood Cell. Mol. Dis. 55(2015) 308-315.

[6] S. Yoshida, T. Kibe, R. Matsubara, S. Koizumi, K. Nara, K. Amano, N. Okumura, Congenital dysfibrinogenemia in a Japanese family with fibrinogen Naples 
(BßAla68Thr) manifesting as superior sagittal sinus thrombosis, Blood Coagul.

Fibrinolysis 28(2017) 580-584.

[7] M. Ikeda, T. Kobayashi, S. Arai, S. Mukai, Y. Takezawa, F. Terasawa, N. Okumura, Recombinant $\gamma$ T305A fibrinogen indicates severely impaired fibrin polymerization due to the aberrant function of hole 'a' and calcium binding sites, Thromb. Res. 134(2014) 518-525.

[8] N. Okumura, K. Furihata, F. Terasawa, R. Nakagoshi, I. Ueno, T. Katsuyama, Fibrinogen Matsumoto I: a gamma 364Asp $\rightarrow$ His (GAT $\rightarrow$ CAT) substitution associated with defective fibrin polymerization, Thromb. Haemost. 75(1996) 887-891.

[9] K. Soya, F. Terasawa, N. Okumura, Fibrinopeptide A release is necessary for effective B:b interactions in polymerization of variant fibrinogens with impaired A:a interactions, Thromb. Haemost. 109(2013) 221-228.

[10] M. Hirota-Kawadobora, S. Kani, F. Terasawa, N. Fujihara, K. Yamauchi, M. Tozuka, N. Okumura, Functional analysis of recombinant B $\beta 15 \mathrm{C}$ and B $\beta 15 \mathrm{~A}$ fibrinogens demonstrates that $\mathrm{B} \beta 15 \mathrm{G}$ residue plays important roles in FPB release and in lateral aggregation of protofibrils, J. Thromb. Haemost. 3(2005) 983-990. 
Table 1 Coagulation tests for plasma

\begin{tabular}{ccccccc}
\hline & $\begin{array}{c}\text { PT } \\
(\%)\end{array}$ & $\begin{array}{c}\text { APTT } \\
(\mathrm{sec})\end{array}$ & \multicolumn{2}{c}{ Fbg $(\mathrm{g} / \mathrm{L})$} & Ratio & $\begin{array}{c}\text { TCT } \\
\text { (sec) }\end{array}$ \\
\cline { 4 - 6 } & & & functional & \multicolumn{2}{c}{ immunological } \\
\hline Proband (Ho) & 33.9 & 43.0 & 1.38 & 3.74 & 0.369 & 90.0 \\
Father (He) & 98.8 & 31.6 & 1.87 & 3.15 & 0.594 & 22.9 \\
Mother (He) & 105.0 & 27.5 & 1.44 & 2.27 & 0.634 & 24.5 \\
Elder sister (He) & 100.8 & 30.0 & 1.93 & 2.88 & 0.671 & 20.6 \\
Younger sister (Ho) & 48.5 & 33.5 & 1.00 & 2.75 & 0.364 & 118.0 \\
Normal range & $70 \sim 130$ & $23 \sim 38$ & $1.80 \sim 3.50$ & $1.80 \sim 3.50$ & $0.80 \sim 1.20$ & $10 \sim 14$ \\
\hline
\end{tabular}

Ho: homozygote, He: heterozygote, TCT: thrombin clotting time 

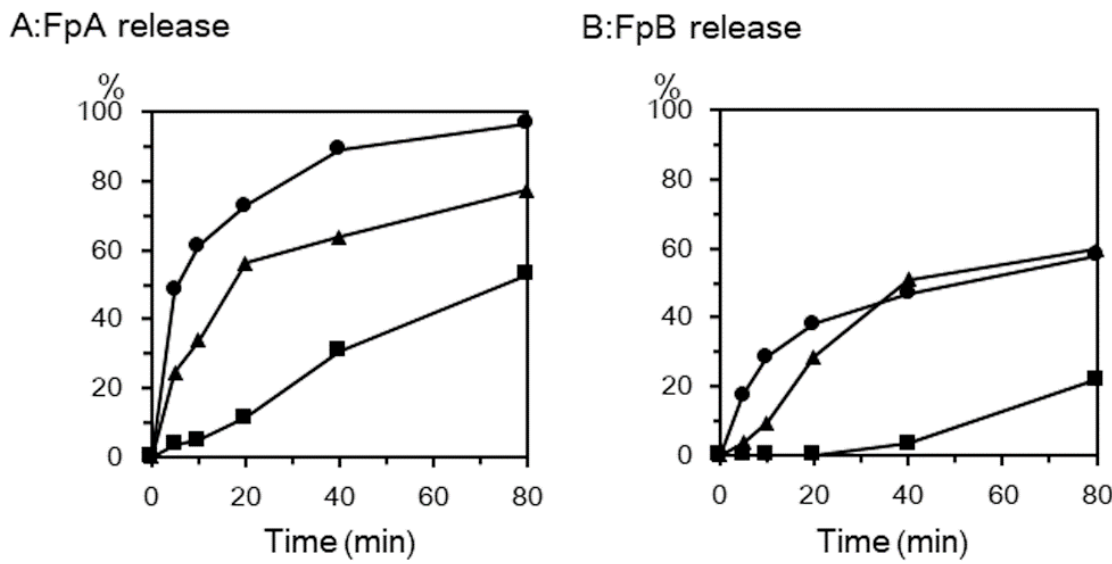

C:Fibrin polymerization

D:Fibrin polymerization
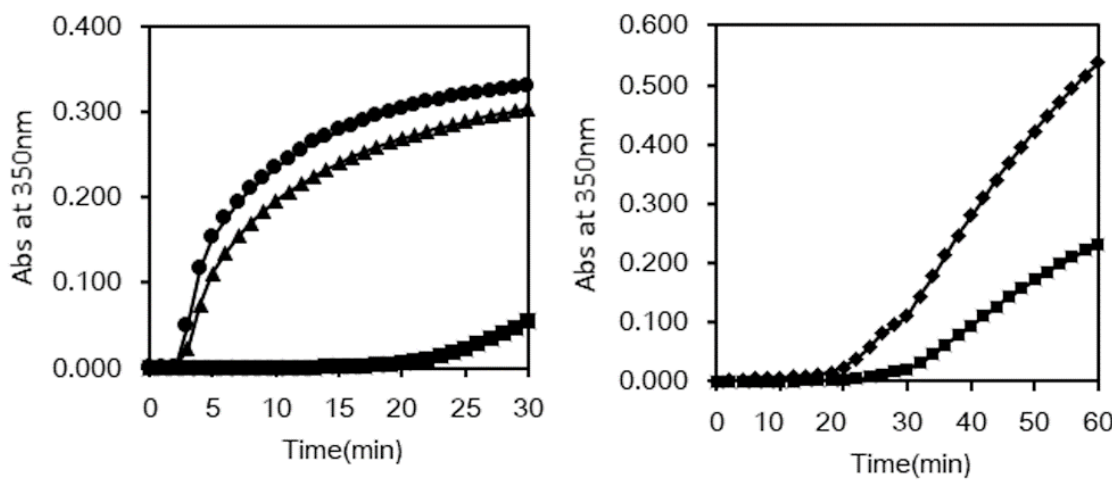

E:Fibrin polymerization

F:FM polymerization
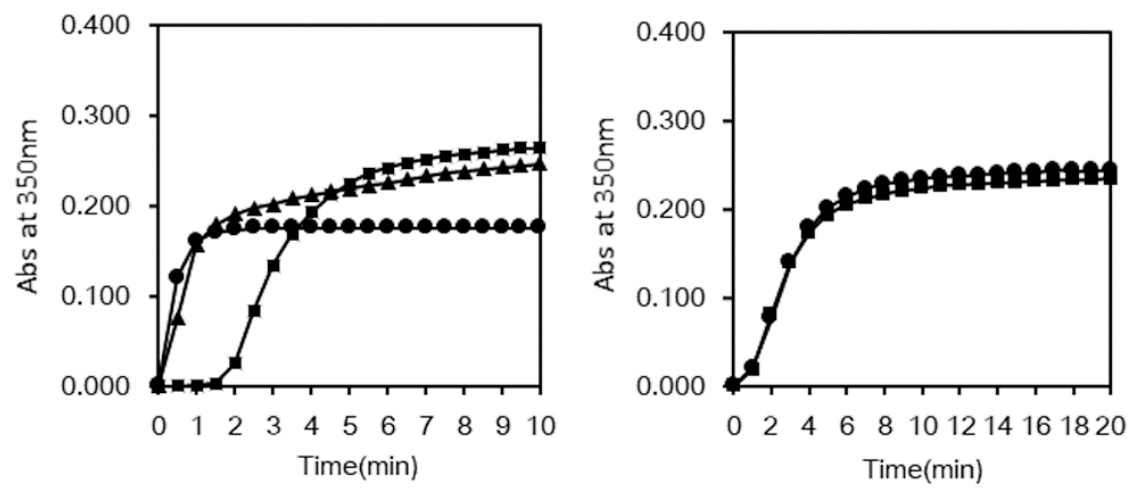


\section{Figure legend}

Figure 1. Thrombin-catalyzed fibrinopeptide release, fibrin polymerization, and fibrin monomer polymerization. Percent fibrinopeptide release curves from a HPLC assay are shown in Panel A: FpA and Panel B: FpB with thrombin $(0.05 \mathrm{U} / \mathrm{ml})$ catalysis. Reactions were run with the wild-type $(\boldsymbol{\bullet})$, father's (heterozygote’s) $(\boldsymbol{\Delta})$, and propositus’ (homozygote’s) ( $\square$ ) fibrinogen $(0.35 \mathrm{mg} / \mathrm{ml})$ at an ambient temperature in HBS. The 100\% release of FpA and FpB was catalyzed with $3.9 \mathrm{U} / \mathrm{mL}$ of thrombin at $37^{\circ} \mathrm{C}$ for 80 minutes.

The thrombin-catalyzed polymerization of fibrinogen $(0.18 \mathrm{mg} / \mathrm{ml})$ was initiated with $0.05 \mathrm{U} / \mathrm{mL}$ of thrombin at an ambient temperature (Panel C). Panel D: The thrombin-catalyzed polymerization of the propositus’ fibrinogen, $0.18(\boldsymbol{\square})$ or 0.36 $\mathrm{mg} / \mathrm{ml}(\diamond)$, was initiated with $0.05 \mathrm{U} / \mathrm{mL}$ of thrombin. Panel E: The thrombin-catalyzed polymerization of fibrinogen $(0.18 \mathrm{mg} / \mathrm{ml})$ was initiated with $1.0 \mathrm{U} / \mathrm{mL}$ of thrombin. Panel F: A total of $0.1 \mathrm{mg} / \mathrm{mL}$ of fibrin monomers (derived from the thrombin-initiated clot) was re-polymerized in pH 7.4 buffer (normal control and the homozygous propositus). Representative turbidity curves from triplicate experiments are shown for the normal control $(\bullet)$, father's (heterozygote) $(\boldsymbol{\Delta})$, and propositus's (homozygote) $(\boldsymbol{\square})$ fibrinogen (Panels C-F). 